\title{
CORRELAÇÃO ENTRE UNIFORMIDADE E NDVI EM POVOAMENTOS DE Tectona grandis L. f.
}

\author{
CORRELATION BETWEEN UNIFORMITY AND NDVI IN STANDS OF Tectona grandis L. $f$.
}

\author{
Lucas Henderson de Oliveira Santos ${ }^{1}$, Gláucia Miranda Ramirez², Márcio William Roque \\ Mariana Peres de Lima Chaves e Carvalho ${ }^{4}$, Lila Mabel Gamarra Ruiz Díaz ${ }^{5}$, \\ Samuel De Pádua Chaves e Carvalho 6
}
1, 2, 3, 4, 5, 6 Universidade Federal de Mato Grosso, Cuiabá, Mato Grosso, Brasil-lucashenderson@ufmt.br, glaucia_ramirez@ufmt.br, roque@ufmt.br,marianaperes@ufmt.br, lilagamarra@ufmt.br\& spccarvalho@ufmt.br

\begin{abstract}
RESUMO
A busca por produtos provindos de Teca é crescente, devido suas características físico-mecânicas. Seu cultivo é cada vez maior em função do valor agregado da madeira. Visando minimizar o ciclo para obtenção de seus produtos, se faz necessário métodos que permitam acompanhar e identificar a qualidade dos plantios. Com isso, o manejo florestal e o sensoriamento remoto auxiliam na seleção de instrumentos de análise para plantios comerciais. $O$ índice de uniformidade auxilia nas tomadas de decisões na qualidade silvicultural dos plantios. Este trabalho buscou correlações entre uniformidade e NDVI para investigar o desenvolvimento da Teca. Foram utilizadas imagens do Landsat8-OLI para obtenção do NDVI e valores de reflectância da Banda 6. A cultura de Teca foi implementada no ano de 2005. Os dados de campo foram provenientes de inventários florestais realizados a cada dois anos a partir do segundo após o plantio. Foram utilizados dados de altura dos inventários executados em maio de 2014 e 2016. Tendo idades de 9 e 11 anos, respectivamente. Os talhões utilizados foram 88, 89, 90, 91, 92 e 93 . Foram mensuradas as alturas das árvores em 47 parcelas com área de aproximadamente $1.551 \mathrm{~m}^{2}$ cada. Posteriormente, foi calculado o índice de uniformidade. A correlação foi feita através do coeficiente de correlação de Pearson. Os resultados do trabalho mostram ser possível acompanhar o desenvolvimento da Teca com a utilização de imagens de satélite em conjunto com dados de inventário florestal.
\end{abstract}

PALAVRAS-CHAVE: Manejo Florestal, NDVI, Sensoriamento Remoto, Uniformidade.

\begin{abstract}
The search for products from Teak is increasing due to its physico-mechanical characteristics. Its cultivation is increasing due to the added value of the wood. In order to minimize the cycle to obtain its products, it is necessary to use methods to monitor and identify the quality of the plantations. With this, forest management and remote sensing aid in the selection of analysis instruments for commercial plantations. The uniformity index assists decision making in the silvicultural quality of plantations. This work sought correlations between uniformity and NDVI to investigate the development of Teak. Landsat8-OLI images were used to obtain the NDVI and Band 6 reflectance values. The Teca culture was implemented in the year 2005. Field data were obtained from forest inventories carried out every two years from the second after the planting. Height data were taken from the inventories made in May 2014 and 2016. They were 9 and 11 years old respectively. The plots used were 88, 89, 90, 91, 92 and 93 . The tree heights were measured in 47 plots with an area of approximately $1,551 \mathrm{~m}^{2}$ each. Subsequently, the uniformity index was calculated. Correlation was done using Pearson's correlation coefficient. The results of the work show that it is possible to monitor the development of Teak with the use of satellite images in conjunction with forest inventory data.
\end{abstract}

KEYWORDS: Forest Management, NDVI, Remote Sensing, Uniformity. 


\section{INTRODUÇÃO}

A Teca possui um quantitativo expressivo em área plantada no mundo. Mundialmente, a área plantada com a espécie excede 6,8 milhões de hectares (MIDGLEY et al., 2015). Sua madeira é de alto valor no mercado internacional, no qual são registrados preços mais elevados que o do Mogno. Tal fator favorece o mercado produtor brasileiro, pois é possível cultivar essa espécie em menor tempo e com alta qualidade (FIGUEIREDO et al., 2005).

Realizar práticas de manejo florestal é de fundamental importância, pois influenciam no aumento da produtividade da floresta, uma vez que pequenos desvios podem ocasionar grandes perdas na produção. O método mais utilizado de se estimar a produtividade madeireira é por meio de inventários florestais (CAMPOS \& LEITE, 2013). Nos inventários são coletados dados como DAP (diâmetro à altura do peito), altura dos indivíduos, espessura da casca, número de árvores mortas, ataque de pragas ou doenças, dentre outras informações que contribuam para o monitoramento e qualidade silvicultural.

A uniformidade de plantações florestais tem sido recentemente utilizada como ferramentas de qualidade. Também permitem decisões mais assertivas, visto que estudos mostraram a melhoria das funções de produção quando considerada essa informação (HAKAMADA et al., 2015). A uniformidade é expressa pelo índice de uniformidade que permite selecionar indivíduos que melhor se desenvolveram. Portanto, quanto mais uniforme o crescimento, maior é a homogeneidade da floresta. Há poucos registros de investigações, principalmente com Teca, que comprovem se a uniformidade ao longo do ciclo de cultivo possui relação direta com a produtividade final (LITTLE et al., 2003).

O monitoramento de povoamentos florestais também pode ser realizado a partir de técnicas de sensoriamento remoto (SR). Alguns índices de vegetação gerados a partir de imagens de satélites podem ser utilizados para estimar o desenvolvimento da cultura, como NDVI (índice da diferença normalizada), SAVI (índice de vegetação ajustado ao solo) e GVI (índice de vegetação verde) (AVERY \& BERLIN, 1992; MENESES, 2001).

Utilizar índices obtidos por meio de SR em plantios de Teca pode tornar menos oneroso o tempo gasto nos inventários tradicionais, trazer agilidade aos processos de monitoramento da cultura e ser decisivo na tomada de decisões durante o manejo da espécie.

Em estudos com Eucalipto, o SR tem proporcionado dados de grande valia para cultura, por isso é importante avaliar a eficácia dessas informações na cultura da Teca. Também são utilizados procedimentos tradicionais para obtenção de volume de madeira, aliados as técnicas e ferramentas de sensoriamento (WATZLAWICK, 2009). Os índices de vegetação também são usados no auxílio de estimativa do volume de povoamentos de eucalipto (LEAL et al., 2013).

Portanto, devido à grande importância comercial dessa espécie, buscar correlações entre os índices de sensoriamento remoto e uniformidade se tornam imprescindíveis como ferramenta para obtenção de subsídios no estabelecimento de estratégias para melhorar a produção.

Este trabalho teve como objetivo avaliar a correlação entre os índices de uniformidade com dados de imagens do satélite Landsat 8, a fim de inferir sobre o desenvolvimento de povoamentos de Teca.

\section{MATERIAL E MÉTODOS}

\section{Área de estudo}

Os dados que compuseram este estudo foram obtidos em plantações clonais de Teca localizadas na região sudoeste do estado de Mato grosso (Figura 1).

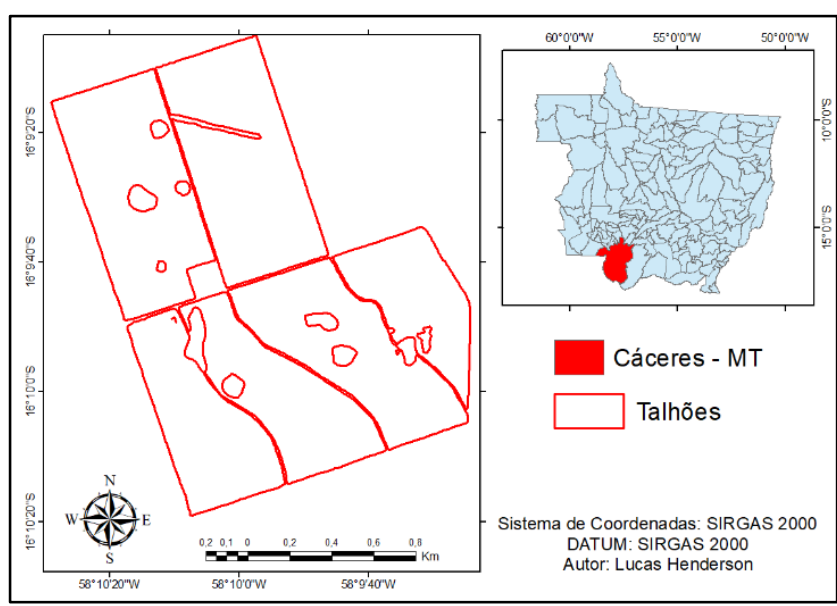

Figura 1. Localização da área de estudo.

A região apresenta altitude média de $150 \mathrm{~m}$, temperatura de $25,4^{\circ} \mathrm{C}$, pluviosidade total anual com variação entre $1.300 \mathrm{~mm}$ e $1.400 \mathrm{~mm}$ (SEPLAN, 2011). Segundo a classificação de Köppen, o clima da região é classificado como Aw, com verão chuvoso e período de seca no inverno (ALVARES et al., 2013).

\section{Dados de campo}

O povoamento foi implantado no ano de 2005 . Os 
dados de campo foram provenientes de inventários realizados a cada dois anos a partir do segundo ano do plantio. Foram utilizados valores das alturas obtidas dos inventários feitos em maio de 2014 e 2016, que corresponderam as idades de 9 e 11 anos, respectivamente. Nesses, foram amostrados seis talhões (Figura 1) com 47 parcelas dispostas sistematicamente.

Para o cálculo do índice de uniformidade, foi adaptada a metodologia utilizada por Hakamada (2012) em plantios clonais de Eucalyptus spp., o qual utilizou-se do índice PV50 sugerido por Stape et al. (2006) para volume. O índice foi obtido por meio de dados altura total das árvores expresso em metros e chamado de PH50.

O PH50 é a porcentagem de altura acumulada das 50\% menores árvores da parcela, ranqueadas em ordem crescente e obtido conforme a Equação 1.

$$
P H 50_{j k}=\frac{\sum_{i=1}^{\frac{n}{2}} H T_{i j k}}{\sum_{i=1}^{n} H T_{i j k}}
$$

Em que: $P H 50_{j k}=$ porcentagem acumulada da altura individual das $50 \%$ menores árvores plantadas na parcela $j$, na idade $k ; H T=$ altura individual da árvore $i$, na parcela $j$, na idade $k$; e $n=$ número de observações.

Neste estudo, o PH50 é também o índice de uniformidade. A representação gráfica ilustra o cálculo do índice (Figura 2).

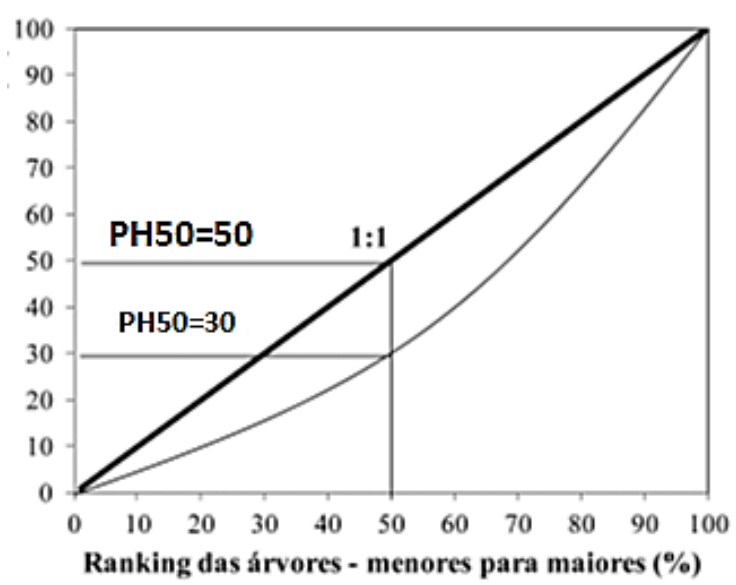

Figura 2. Representação gráfica do PH50 (adaptado de Hakamada, 2012).

No eixo $X$ são ranqueadas as árvores da menor para a maior altura e no eixo $Y$ são inseridos os valores de altura. A linha em negrito representa a linha da igualdade, onde $50 \%$ das árvores seriam responsáveis por 50\% da altura da parcela. A linha preta representa um exemplo hipotético, onde $50 \%$ das árvores correspondem a apenas 30\% da altura da parcela.

Os cálculos foram realizados para cada parcela e, posteriormente, as médias por talhão foram calculadas. Para validação do índice de uniformidade, fez-se a determinação do intervalo ótimo de uniformidade (IOU), a fim de avaliar o comportamento da uniformidade de acordo com o potencial produtivo do sítio. Para a avaliação em escala espacial e temporal, utilizou-se da rede de inventário florestal para capturar a evolução da uniformidade do povoamento.

O intervalo ótimo de uniformidade é o parâmetro para indicação de máxima uniformidade e o mínimo necessário para ser considerado uniforme. O limite superior do IOU é de $50 \%$ e o limite inferior é representado pela média do PH50 subtraída do desvio padrão (Equação 2), para que o IOU representasse mais de $95 \%$ das alturas contidas nos testes clonais.

$$
I O U=50 \%>I O U_{i j}>\bar{X}_{i j}-1 x S_{i j}
$$

Em que: $I O U_{i j}=$ intervalo ótimo de uniformidade da parcela $i$ na idade $j ; \bar{X}_{i j}=$ média do PH50 da parcela $i$ na idade $j$; e $S_{i j}=$ desvio padrão da média do PH50 da parcela $i$ na idade $j$.

\section{Imagem do satélite LANDSAT8/OLI}

Foram utilizadas imagens do LANDSAT8/OLI, órbita/ponto 228/71, referentes ao mês de maio de 2014 e 2016. Essas datas foram as escolhidas por conter dados do inventário florestal e por não apresentar nuvens.

Foram utilizadas as bandas 4 (vermelho), 5 (infravermelho próximo) e 6 (banda do infravermelho médio) com $30 \mathrm{~m}$ de resolução espacial. As imagens de refletância de superfície foram disponibilizadas por United States Geological Survey (USGS).

Os produtos de refletância de superfície fornecem uma estimativa da reflectância espectral de superfície medida no nível do solo na ausência de espalhamento ou absorção atmosférica, com minimização dos efeitos da geometria de iluminação.

Os dados Landsat 8 Reflectance foram gerados a partir de códigos de reflexão da superfície Landsat (LaSRC), o qual faz uso da banda 1 (aerossol costal) para obter os dados de aerossóis, usa dados climáticos do satélite MODIS e um modelo de transferência radiativa exclusivo. Além disso, codifica o ângulo zenital de visão para $0^{\circ}$, diminuindo a influência do ângulo zenital solar. Esses dados também são usados como parte do cálculo da correção atmosférica. 
Para cada pixel na imagem, foi calculado os valores do NDVI a partir das intensidades de reflexão dos canais do infravermelho próximo e vermelho do próprio pixel, de acordo com a Equação 3.

$$
N D V I=\frac{\mathrm{IVP}-\mathrm{V}}{\mathrm{IVP}+\mathrm{V}}
$$

Em que: $N D V I$ = índice de vegetação por diferença normalizada; $I V P=$ corresponde a banda espectral do infravermelho próximo; e $V=$ corresponde a banda espectral do vermelho.

Também foram coletados os valores de reflectância dos pixels presentes na banda 6 , de acordo com as áreas amostrais alocadas.

\section{Obtenção das amostras nas imagens}

Para a obtenção dos valores de reflectância das imagens do satélite Landsat 8 , as amostras foram alocadas ao entorno da coordenada central em cada parcela utilizada do inventário, onde foram coletados 4 pixels por amostra, num total de 47 amostras.

Para o cálculo de correlação, foi utilizado os valores de reflectância dos quatro pixels amostrados e então calculadas as médias por talhão.

\section{Análise de correlação}

A avaliação do NDVI com o índice de uniformidade foi feita de acordo com a correlação de Pearson, a qual foi calculada para quantificar a intensidade da associação linear existente entre as variáveis obtendo-se um índice sem dimensão.

O mesmo é situado entre - 1 e 1 que reflete a extensão de uma relação linear entre dois conjuntos de dados, através do programa EXCEL, para determinação do coeficiente de correlação de Pearson (nível de significância igual a 95\%) entre os valores de reflectância e os parâmetros biofísicos obtidos em campo. Calculados conforme a Equação 4.

$$
r=\frac{\sum_{i=1}^{n}\left(x_{i}-\bar{x}\right)\left(y_{i}-\bar{y}\right)}{\sqrt{\left[\sum_{i=1}^{n}\left(x_{i}-\bar{x}\right)^{2}\right]\left[\sum_{i=1}^{n}\left(y_{i}-\bar{y}\right)^{2}\right]}}
$$

Em que: $\bar{x}$ e $\bar{y}=$ respectivamente as médias dos valores $x_{i}$ e $y_{i}$.

Neste estudo foi avaliada a correlação entre NDVI (índice da diferença normalizada), valores de Reflectância da Banda 6 do sensor OLI presente no Landsat 8 que são dados gerados a partir de imagens de satélite com o IU (índice de uniformidade) que é obtido a partir de dados de inventário florestal.

Os dados foram interpretados a partir da análise de correlação proposta por SANTOS (2007).

\section{RESULTADOS E DISCUSSÃO}

\section{Valores médios das variáveis}

A Tabela 1, referente aos anos de 2014 e 2016, apresenta os valores médios de NDVI, Valores de reflectância em porcentagem da banda 6 e índice de uniformidade utilizados como entrada na análise de correlação de Pearson.

Tabela 1: Valores médios de NDVI, banda 6 e índice de uniformidade dos anos de 2014 e 2016.

\begin{tabular}{ccccc}
\hline Talhões & NDVI & Banda 6 (\%) & IU & Ano \\
\hline $\mathbf{8 8}$ & 0,827 & 14,03 & 0,48 & 2014 \\
$\mathbf{8 9}$ & 0,769 & 16,81 & 0,47 & 2014 \\
$\mathbf{9 0}$ & 0,835 & 14,49 & 0,47 & 2014 \\
$\mathbf{9 1}$ & 0,829 & 14,24 & 0,47 & 2014 \\
$\mathbf{9 2}$ & 0,792 & 15,73 & 0,43 & 2014 \\
$\mathbf{9 3}$ & 0,847 & 13,63 & 0,47 & 2014 \\
$\mathbf{8 8}$ & 0,854 & 15,89 & 0,47 & 2016 \\
$\mathbf{8 9}$ & 0,782 & 19,61 & 0,44 & 2016 \\
$\mathbf{9 0}$ & 0,817 & 17,68 & 0,44 & 2016 \\
\hline $\mathbf{9 1}$ & 0,812 & 16,85 & 0,45 & 2016 \\
\hline $\mathbf{9 2}$ & 0,810 & 17,15 & 0,46 & 2016 \\
\hline 93 & 0,792 & 17,40 & 0,46 & 2016 \\
\hline
\end{tabular}

Em que: IU = índice de uniformidade; e NDVI = índice de vegetação da diferença normalizada.

Hakamada (2012), estudando a redução média do índice de uniformidade com a utilização a variável volume, observou redução de $15,1 \%$ em uniformidade e de IMA (Incremento médio anual) de 9,6 $\mathrm{m}^{3} \mathrm{ha}^{-1} \mathrm{ano}^{-1}$. Assim, pode-se inferir que houve uma perda de $0,63 \mathrm{~m}^{3} \mathrm{ha}^{-1}$ ano ${ }^{1}$ no IMA para cada $1 \%$ de redução do PV50.

Para a Teca, observa-se que a redução de uniformidade faz com que a reflectância de NDVI diminua e o desvio padrão das alturas aumente. Todavia, vale ressaltar que o método NDVI não pode ser considerado determinístico e o único parâmetro a ser utilizado para estudos sobre alterações ocorridas em áreas de cobertura vegetal (VELASCO et al., 2007). 


\section{Determinação do intervalo ótimo de uniformidade e índice de uniformidade}

A partir dos cálculos, o intervalo ótimo estabelecido para os dois anos foi de 0,44. Apenas o talhão 92 no ano de 2014 não esteve dentro do intervalo ótimo de uniformidade. Considerando a média dos talhões dos valores de uniformidade em porcentagem, houve um decréscimo de $2,22 \%$ de uniformidade, sendo que não houve diferença estatisticamente significativa entre os valores médios do índice de uniformidade para os anos de 2014 e 2016.

Em cultivos florestais, para se aumentar ou manter o nível de uniformidade do povoamento, são utilizadas técnicas de manejo florestal, principalmente com tratos silviculturais. Assim, é possível aumentar a disponibilidade de recursos de crescimento, como água, luz e nutrientes (NYLAND, 2002).

Hakamada (2012), em estudos utilizando redes de inventário florestal para monitoramento da qualidade de plantios de eucalipto, observou a ausência de diferença de uniformidade entre os testes clonais, mostrando que, quando as recomendações técnicas e a qualidade da execução das operações são realizadas dentro de um padrão ótimo, a uniformidade possui um comportamento padrão independentemente da capacidade produtiva do sítio.

Aspinwall et al. (2011), em estudos sobre influência do nível de melhoramento genético na uniformidade e produtividade de povoamentos de Pinus taeda no sudeste dos Estados Unidos, concluíram que a uniformidade em povoamentos clonais é dependente da qualidade silvicultural. A partir disso, foi possível inferir que, mesmo as árvores sendo geneticamente idênticas, não se pode garantir a elevada uniformidade.

Boyden et al. (2008), em seus estudos de comparação de produtividade de clones de Eucalyptus spp. utilizandose de plantios seminais, sugeriram que a maior produtividade dos povoamentos clonais se deu pela maior uniformidade do povoamento, permitindo que a eficiência do uso de recursos fosse elevada árvore-a-árvore.

\section{Índice de vegetação da diferença normalizada}

Os resultados dos índices de vegetação estão apresentados na Figura 3.

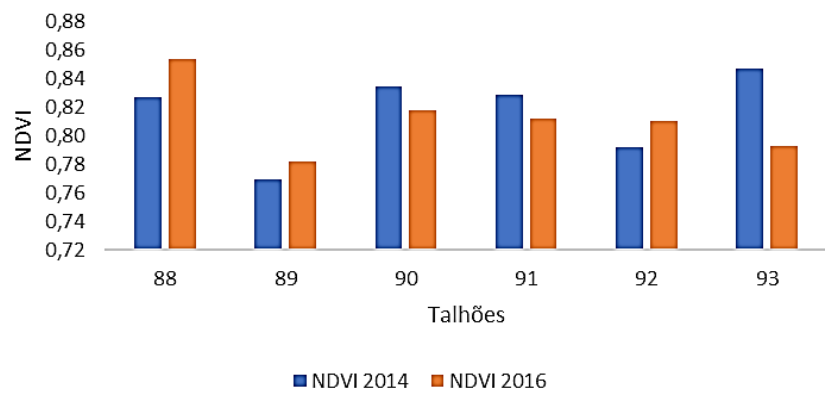

Figura 3. Valores de NDVI dos anos de 2014 e 2016.

A partir dos valores de reflectância da imagem de satélite, foram obtidos os valores de NDVI. As médias obtidas foram de 0,8168 e 0,8116 para os anos de 2014 e 2016, respectivamente. É possível observar que, com a redução da uniformidade de $2,22 \%$, há uma queda do NDVI, mostrando que ele é sensível a obtenção de valores de reflectância com relação ao dossel de plantios clonais de Teca.

Para o NDVI, quanto mais próximo de 1 , maior é a atividade vegetativa no local representado pelo pixel. Valores negativos ou próximos de zero indicam áreas de água, edificações ou solo nu, onde há pouca ou nenhuma atividade clorofiliana (ROSENDO, 2005).

Em comparação com outras espécies, como o Eucalyptus grandis x urophylla, Almeida et al. (2015) encontraram valores de NDVI que variaram entre 0,33 e 0,79 com idades entre 0,5 e 7 anos. Tal aumento explica-se devido ao Eucalipto apresentar um crescimento mais acelerado em relação a Teca. Logo, com o aumento de crescimento de área foliar, a espécie estudada aumenta os valores de NDVI.

Estima-se que com a Teca ocorra o mesmo processo e que, com o avanço em crescimento, os valores de NDVI também aumentarão e elevando, consequentemente, sua correlação em relação aos parâmetros estudados, como altura e banda 6 .

As imagens Landsat, ainda que muito utilizadas, principalmente em função da sua série temporal, podem ter sua eficácia limitada devido sua resolução espacial de $30 \mathrm{~m}$, o que implica em mistura espectral dos alvos dentro dos pixels (RUDORFF, 2006; MELLO et al., 2012). Sendo assim, é sugestivo estudos com outras resoluções espaciais para averiguar a diferença entre os produtos. Para isso, pode-se citar exemplos como o novo satélite Sentinel-2, com resolução espacial de $10 \mathrm{~m}$ ou CCD/CBERS com $20 \mathrm{~m}$. Ide (2017), em estudos de identificação em áreas irrigadas, utilizaram de imagens do Sentinel-2/MSI e CBERS 4, onde foram encontrados valores de classificação considerados como sendo excelentes. 


\section{Valores de reflectância da banda 6}

Os valores médios de reflectância em porcentagem obtidos nos anos de 2014 e 2016 foram respectivamente $14,78 \%$ e $17,43 \%$.

Ponzoni \& Disperati (1994), trabalhando com caracterização espectral de estágios sucessionais de vegetação encontraram no infravermelho médio valores de reflectância entre 15 e $18 \%$ da banda 6 . Correspondendo com o encontrado, uma vez que a maior quantidade de folhas existentes nos estágios mais avançados reduz a reflectância do dossel pela maior oferta de água, que nessa faixa espectral domina o comportamento espectral da vegetação. As imagens foram capturadas em maio, depois do período de chuvas, onde a planta ainda está com suas folhas (GOEL, 1988).

No infravermelho médio, é esperado que à medida em que se verifica uma maior perda de uniformidade do dossel, sua reflectância nessa região espectral deva decrescer, devido ao sombreamento mútuo (BERNARDES, 1996; SOUSA, 1996; MORAN et al., 2000).

\section{Correlação de Pearson}

Na Tabela 2 estão presentes os valores de correlação de Pearson dos anos de 2014 e 2016, em conjunto com a interpretação.

Tabela 2. Correlação entre valores médios de NDVI, banda 6 e índice de uniformidade para Teca.

\begin{tabular}{|c|c|c|c|c|}
\hline Correlações & 2014 & Resultado & 2016 & Resultado \\
\hline NDVI & 0,782 & 19,61 & 0,440 & 2,430 \\
\hline Banda 6 & 0,407 & $\begin{array}{c}\text { Fraca } \\
\text { positiva }\end{array}$ & 0,597 & $\begin{array}{c}\text { Moderada } \\
\text { positiva }\end{array}$ \\
\hline
\end{tabular}

As relações inversas são esperadas nas regiões do visível e do infravermelho próximo, principalmente na vegetação de porte herbáceo ou arbustivo. Contudo, nessa última região espectral há de serem considerados os aspectos referentes ao regime de sombreamento mútuo discutido por Ponzoni (1993), Bernardes (1996) e Sousa (1996), que nas formações arbóreas acabam também invertendo essa relação.

Ponzoni \& Rezende (2004) observaram que os valores mais elevados de qualquer parâmetro biofísico proporcionaram estimativas mais próximas da realidade. Logo, valores de reflectância de superfície não são sensíveis a detecção de pequenos valores biofísicos.

Entre os dados de altura do dossel com os dados radiométricos das bandas do infravermelho médio, foi observado a possibilidade de se obter relações entre resultados a partir dos dados provenientes das bandas espectrais do visível e do infravermelho próximo. Tal fato redireciona os esforços na elaboração de índices que costumeiramente são estabelecidos com dados das bandas do vermelho e do infravermelho próximo (PONZONI \& REZENDE, 2004).

\section{CONCLUSÕES}

Quanto menor a uniformidade do estrato florestal, menor é o NDVI, cuja correlação é inversamente proporcional. Assim, quanto maior o desvio padrão das alturas, menores são os valores de refletância obtidos nos pixels das amostras.

O índice de uniformidade possui correlação positiva com o NDVI, em que, quanto mais uniforme o plantio, maiores são os valores de reflectância obtidos nos pixels utilizados para o cálculo do NDVI.

\section{REFERÊNCIAS}

ALMEIDA, A. et al. Índice de Área Foliar de Eucalyptus Estimado por Índices de Vegetação Utilizando Imagens TM - Landsat 5. Floresta e Ambiente, v.22, n.3, p.368-376, 2015.

ASPINWALL, J.M. et al. Genetic effects on stand-level uniformity and above- and belowground dry mass production in juvenile loblolly pine. Forest Ecology and Management, v.262, p.609-619, 2011.

ALVARES, C.A. et al. Köppen's climate classification map for Brazil. Meteorologische Zeitschrift, v.22, n.6, p.711-728, 2013.

AVERY, T.E.; BERLIN, G.L. Fundamentals of Remote sensing and airphoto interpretation. Prentice Hall, Upper Saddle River, p. 472, 1992.

BERNARDES, S. Índices de vegetação e valores de proporção na caracterização de floresta tropical primária e estádios sucessionais na área de influência da Floresta de Tapajós-Estado do Pará. 1996. 94p. (Dissertação de mestrado).

BOYDEN, S. et al. Competition among trees depends on genetic variation and resource supply. Ecology, v.89, p.2850-2859, 2008.

CAMPOS, J.C.C.; LEITE, H.G. Mensuração florestal: perguntas e respostas. 4.ed. Viçosa: UFV, 2013.

FIGUEIREDO, E.O. et al. Teca (Tectona grandis L.F.): principais perguntas do futuro empreendedor florestal. Rio Branco: Embrapa Acre, 2005.2 Disponível em: http://www.cpafac.embrapa.br/pdf/doc97.pdf

GOEL, N.S. Models of vegetation canopy reflectance and their use in estimation of biophysical parameters from reflectance data. Remote Sensing Reviews, v.4, p.1-21, 1988. 
HAKAMADA, R.E. et al. Uso do inventário florestal e da uniformidade entre árvores como ferramenta de monitoramento da qualidade silvicultural em plantios clonais de eucalipto. Scientia Forestalis, v.43, n.105, p.27-39, 2015.

HAKAMADA, R.E. Uso do inventário florestal como ferramenta de monitoramento da qualidade silvicultura em povoamentos clonais de Eucalyptus. 2012. 114p. (Dissertação de mestrado).

IDE, A.K.; ANDRADE, M.C.K. Impactos da perenização do Riacho Terra Nova na agricultura irrigada do município de Cabrobó/PE. Meio Ambiente E Sustentabilidade, v.10, n.6, p.57-77, 2017.

LEAL, F.A. et al. Índice de vegetação na estimativa do volume em um povoamento de eucalipto. Enciclopédia biosfera, v.9, n.17, p.1638, 2013.

LITTLE, K.M. et al. Eucalyptus grandis x E. camaldulensis variability and intra-genotypic competition as a function of different vegetation management treatments. New Forests, v.25, p.227242, 2003.

MENESES, P.R., NETTO, J.S.M. Sensoriamento remoto: reflectância dos alvos naturais. Brasília: Editora Universidade de Brasília/Embrapa Cerrados, 2001.

MELLO, I.A.Y. et al. Avaliação de técnicas de classificação digital de imagens Landsat em diferentes padrões de cobertura da terra em Rondônia. Revista Árvore, v.36, n.3, p.537-547, 2012.

MIDGLEY, S. et al. Planted teak: global production and markets, with reference to Solomon Islands. ACIAR Technical Reports 85. Canberra: Australian Centre for International Agricultural Research, 2015.2 Disponível em: https://www.aciar.gov.au/file/81926/download?token=LrWrS8a $\mathrm{x}$.

MORAN, E.F. et al. Effects of soil fertility and land-use on forest successional in Amazônia. Forest Ecology and Management, v.139, p.93-108, 2000.

NYLAND, R.D. Silviculture: concepts and applications. Long Grove: Waveland press. 2.ed. 2002.

PONZONI, F.J. Aplicação do modelo SAIL no estudo da reflectância espectral de dosséis de mudas de Eucalyptus sp. e de Aspidosperma sp. 1993. 253p. (Tese de doutorado).

PONZONI, F.J.; DISPERATI, A.A. Aplicação do modelo SAIL no estudo da reflectância espectral de dosséis de mudas de Eucalyptus sp. e de Aspidosperma sp. Revista Floresta, v.21, n.1/2, p.45-70, 1994.

PONZONI, F.J.; REZENDE, A.C.P. Caracterização espectral de estágios sucessionais de vegetação secundária arbórea em Altamira (PA), através de dados orbitais. Árvore, v.28, n.4, p.535545,2004

ROSENDO, J.S. Índices de vegetação e monitoramento do uso do solo e cobertura vegetal na Bacia do rio Araguari -MG utilizando dados do sensor Modis. 2005. 130p. (Dissertação de mestrado).

RUDORFF, B.F.T. Produtos de sensoriamento remoto. INPE:
Programa Educa Sere, 2006. Disponível em: http://www3.inpe.br/unidades/cep/atividadescep/educasere/in dex.htm.

SANTOS, C. Estatística descritiva: manual de auto-aprendizage. Lisb Ed., 2007.

SEPLAN - SECRETARIA DE PLANEJAMENTO E COORDENAÇÃO DO ESTADO DE MATO GROSSO. Clima, análise e representação cartográfica. Ed. Entrelinhas, 2011.

SOUSA, C.L.; PONZONI, F.J. Considerações sobre transformação de dados orbitais para estimativas do Índice de Área Foliar (IAF) 80 Simpósio Brasileiro de Sensoriamento Remoto, 1996.

STAPE, J. L et al. Indicadores de qualidade silvicultural na Aracruz: 2000 a 2005. Piracicaba: IPEF, 2006.

VELASCO, G.D.N. et al. Aplicação do índice de vegetação NDVI (Normalized Difference Vegetation Index) em imagens de alta resolução no município de São Paulo. Revista da Sociedade Brasileira de Arborização Urbana, v.2, n.3, p.1-12, 2007.

WATZLAWICK, L.F. et al. Estimativa de biomassa e carbono em floresta com araucária utilizando imagens do satélite IKONOS II. Ciência Florestal, v.19, p.169-181, 2009. 